OPEN ACCESS

Edited by:

Gilles Blancho,

University of Nantes, France

Reviewed by:

Vokaer Benoit,

Free University of Brussels, Belgium

Philippe Saas,

Etablissement Français

du Sang BFC, France

*Correspondence:

Jeanne E. Hendrickson

jeanne.hendrickson@yale.edu

Specialty section:

This article was submitted to Alloimmunity and Transplantation,

a section of the journal

Frontiers in Immunology

Received: 20 March 2017

Accepted: 17 July 2017

Published: 07 August 2017

Citation:

Natarajan P, Liu D, Patel SR, Santhanakrishnan M, Beitler D, Liu J, Gibb DR, Liepkalns JS, Madrid DJ,

Eisenbarth SC, Stowell SR and

Hendrickson JE (2017) CD4

Depletion or CD4OL Blockade Results

in Antigen-Specific Tolerance

in a Red Blood Cell

Alloimmunization Model.

Front. Immunol. 8:907.

doi: 10.3389/fimmu.2017.00907

\section{CD4 Depletion or CD40L Blockade Results in Antigen-Specific Tolerance in a Red Blood Cell Alloimmunization Model}

\author{
Prabitha Natarajan', Dong Liu', Seema R. Patel ${ }^{2}$, Manjula Santhanakrishnan', \\ Daniel Beitler ${ }^{1}$, Jingchun Liu' ${ }^{1}$, David R. Gibb' ${ }^{1}$, Justine S. Liepkalns', \\ David J. Madrid ${ }^{3}$, Stephanie C. Eisenbarth ${ }^{1,4}$, Sean R. Stowell ${ }^{2}$ and \\ Jeanne E. Hendrickson ${ }^{1,3 *}$
}

${ }^{1}$ Department of Laboratory Medicine, Yale University School of Medicine, New Haven, CT, United States, ${ }^{2}$ Department of Pathology and Laboratory Medicine, Emory University School of Medicine, Atlanta, GA, United States, ${ }^{3}$ Department of Pediatrics, Yale University School of Medicine, New Haven, CT, United States, ${ }^{4}$ Department of Immunobiology, Yale University School of Medicine, New Haven, CT, United States

Approximately $3-10 \%$ of human red blood cell (RBC) transfusion recipients form alloantibodies to non-self, non-ABO blood group antigens expressed on donor RBCs, with these alloantibodies having the potential to be clinically significant in transfusion and pregnancy settings. However, the majority of transfused individuals never form detectable alloantibodies. Expanding upon observations that children initially transfused with RBCs at a young age are less likely to form alloantibodies throughout their lives, we hypothesized that "non-responders" may not only be ignorant of antigens on RBCs but instead tolerized. We investigated this question in a reductionist murine model, in which transgenic donors express the human glycophorin A (hGPA) antigen in an RBC-specific manner. Although wild-type mice treated with poly IC and transfused with hGPA RBCs generated robust anti-hGPA IgG alloantibodies that led to rapid clearance of incompatible RBCs, those transfused in the absence of an adjuvant failed to become alloimmunized. Animals depleted of $\mathrm{CD}^{+}$cells or treated with CD40L blockade prior to initial hGPA $\mathrm{RBC}$ exposure, in the presence of poly IC, failed to generate detectable anti-hGPA IgG alloantibodies. These non-responders to a primary transfusion remained unable to generate anti-hGPA IgG alloantibodies upon secondary hGPA exposure and did not prematurely clear transfused hGPA RBCs even after their CD4 cells had returned or their CD40L blockade had resolved. This observed tolerance was antigen (hGPA) specific, as robust lgG responses to transfused RBCs expressing a third-party antigen occurred in all studied groups. Experiments completed in an RBC alloimmunization model that allowed evaluation of antigen-specific CD4+ ${ }^{+}$-cells (HOD (hen egg lysozyme, ovalbumin, and human duffy $\left.{ }^{b}\right)$ ) demonstrated that CD4OL blockade prevented the expansion of ovalbumin 323-339 specific T-cells after HOD RBC transfusion and also prevented germinal center formation. Taken together, our data suggest that recipients may indeed become tolerized to antigens expressed on RBCs, with the recipient's immune status upon initial 
RBC exposure dictating future responses. Although questions surrounding mechanism(s) and sustainability of tolerance remain, these data lay the groundwork for future work investigating RBC immunity versus tolerance in reductionist models and in humans.

Keywords: red blood cell, alloimmunization, tolerance, CD40L blockade, T-cells

\section{INTRODUCTION}

Transfusion of red blood cells (RBCs) is the most common procedure completed during hospitalizations (1). Although $\mathrm{RBC}$ transfusions are often lifesaving, they are not without risk. In addition to complications such as febrile reactions, bacterial contamination, or infectious disease transmission $(2,3)$, there is a risk of formation of antibodies to non-self blood group antigens ( $\mathrm{RBC}$ alloimmunization). Although transfusions given in non-emergent situations are $\mathrm{ABO}$ and $\mathrm{RhD}$ compatible between donor and recipient, they are typically not matched for other blood group antigens such as $\mathrm{C} / \mathrm{c}, \mathrm{E} / \mathrm{e}, \mathrm{K} / \mathrm{k}$, Jka/Jkb, Fya/Fyb, S/s, etc. Overall there are more than 30 blood groups and hundreds of variants of these groups $(4,5)$.

Red blood cell alloimmunization rates vary depending on patient population studied and antibody detection techniques. As few as $1 \%$ of transfused oncology patients treated with chemotherapy have been described to be alloimmunized, with up to $40-50 \%$ of transfused patients with sickle-cell disease being alloimmunized (6). Because each transfusion exposes a recipient to many non-self blood group antigens, the variation in alloimmunization rates between different patient groups is not fully understood. Despite this exposure to non-self antigens, the majority of transfused individuals never develop RBC alloantibodies. Factors such as the recipient inflammatory status at the time of RBC exposure (7) and underlying autoimmunity (8) are thought to be important variables in the predisposition to develop RBC alloantibodies.

The clinical significance of RBC alloantibodies in transfusion, pregnancy, and transplantation settings cannot be overstated. These antibodies may lead to hemolytic transfusion reactions, which are one of the leading causes of transfusion-associated death reported yearly to the FDA (9). Alloantibodies in those requiring transfusion make locating compatible RBC units difficult and, at times, impossible (10). They contribute to bystander hemolysis in some patients with sickle-cell disease, which can be fatal (11). In a pregnancy setting, RBC alloantibodies may result in hemolytic disease of the newborn (HDFN) $(12,13)$. In a hematopoietic stem cell transplantation setting, these antibodies may impact processing of the graft or engraftment (14), and in a solid organ transplant setting, RBC antibodies against antigens expressed on cells in the kidney (e.g., those in the Kidd family) have been implicated in graft rejection $(15,16)$.

Strategies to prevent RBC alloantibody development are currently limited, with our incomplete understanding of recipient immunologic responses to $\mathrm{RBC}$ transfusion therapy playing a contributing role. The most effective strategy includes transfusion avoidance, which is not feasible in many situations. Another strategy employed includes providing RBC units from donors matching recipients at high risk for alloimmunization at antigens beyond ABO and RhD ("phenotypic matching") (17). However, this strategy is not entirely effective due to the inability to provide $\mathrm{RBCs}$ matched at all antigen sites, due to Rh variant alleles in donors/recipients that may not be appreciated by phenotyping alone $(18,19)$ and due to patients being transfused at multiple different locations including those that may not provide such matched RBCs (20). At the present time, there are no known immunologic or genetic "signatures" of patients at high or low risk of becoming alloimmunized, although the identification of such a signature would be quite valuable (21).

We study murine models of RBC alloimmunization in our laboratory to better understand the immunologic steps resulting in alloantibody formation and to investigate rationale strategies to mitigate the formation of such antibodies (22). Our models allow reductionist studies to be completed that are simply not feasible in humans, including the study of controlled blood group antigenic differences between donor and recipients. Although the ability of a human recipient to respond to a particular antigen on a transfused RBC may depend on the HLA type of the recipient (23), our murine transfusion recipients are MHC identical and thus all capable of presenting the blood group antigens being studied. Further, our models allow for the comparison of single variable changes of the recipient's immune system on alloantibody induction.

In this current study, we investigate the steps leading to an alloimmune response in a model in which murine donor RBCs express the human glycophorin A (hGPA) blood group antigen (24). Others and we have previously observed that transfused hGPA RBCs do not result in an anti-hGPA RBC alloantibody response unless the recipients are treated with an adjuvant around the time of the transfusion or unless the transfused RBCs are coinfused with an adjuvant $(25,26)$. Polyinosinic-polycytidylic acid, a mimetic of viral double-stranded RNA, is one such adjuvant known to enhance RBC alloimmunization in multiple murine models (27). These past data led to the hypothesis that the hGPA $\mathrm{RBC}$ alloimmune response was $\mathrm{CD} 4^{+} \mathrm{T}$-cell dependent and to the thought that co-stimulatory blockade might prevent alloimmunization altogether. Herein, we describe conditions under which immunity versus tolerance can be induced by transfused hGPA $\mathrm{RBCs}$, and we begin to investigate the mechanism(s) involved in these responses.

\section{MATERIALS AND METHODS}

\section{Mice}

$\mathrm{FVB} / \mathrm{NCr}$ mice were purchased from Charles River Laboratories (Wilmington, MA, USA). C57BL/6 mice were purchased from 
the National Cancer Institute (Frederick, MD, USA) or Taconic (Hudson, NY, USA). Transgenic mice expressing hGPA (24) on the RBCs on FVB genetic background (H2-q) generously provided by the New York Blood Center and HOD mice that have RBC-specific expression of hen egg lysozyme, ovalbumin, and human Duffy b (28) or the KEL glycoprotein (29) at a high density ("KEL hi") were bred in a non-pathogen-free facility at Yale University. OTII mice on a CD45.1 C57BL/6 (H2b) background whose $\mathrm{CD}^{+}{ }^{+} \mathrm{T}$-cells have transgenic T-cell receptors specific for ovalbumin were also bred at Yale. Transfusion-recipient mice were $8-12$ weeks of age and on FVB background unless specified otherwise. All procedures and protocols were approved by Emory and Yale University's Institutional Animal Care and Use Committee.

\section{CD4 Depletion and CD40L Blockade}

For CD4 depletion experiments, a previously optimized dosing schedule was utilized (30): mice were given two IP injections of $200 \mu \mathrm{g}$ anti-mouse CD4 monoclonal antibody (clone: GK1.5, BioXcell, West Lebanon, NH, USA), saline, or an isotype-matched control 2 days apart. $48 \mathrm{~h}$ after the second injection, mice were transfused. On day 7 post-transfusion, another injection of $300 \mu \mathrm{g}$ GK1.5 antibody was given.

For CD40L blocking experiments, another previously optimized dosing schedule was utilized (31): all mice were given IP injections of $250 \mu \mathrm{g}$ anti-mouse CD40L(CD154) monoclonal antibody (clone: MR1, BioXcell, West Lebanon, NH, USA), saline, or an isotype-matched control every 2 days starting on the day of transfusion until 6 days after the transfusion. Additional MR1 was also given on days 9 and 12 .

\section{Transfusion}

Mice were transfused (IV injection) with $75 \mu \mathrm{L}$ of fresh packed transgenic hGPA or HOD RBCs that had been collected in the anticoagulant preservative solution CPDA-1 (citrate phosphorus dextrose adenine, Jorgensen Labs, Henry Schein, Melville, NY, USA) and filter leukoreduced over a Pall (East Hills, NY, USA) syringe filter. Some recipients were IP injected with $100 \mu \mathrm{g}$ poly IC, polyinosinic-polycytidylic acid (Invivogen, San Diego, CA, USA), approximately 3-4 h before transfusion. FVB recipient mice were used for hGPA experiments as the hGPA donors are on an FVB background, and C57BL/6 mice were used for HOD experiments as the HOD donors are on a C57BL/6 background.

\section{Fluorescent Labeling for RBC Clearance}

After collection and leukoreduction, donor hGPA or wild-type FVB/NCr RBCs were labeled with chloromethylbenzamido $1,1^{\prime}$-dioctadecyl-3,3,3',3'-tetramethylindocarbocyanine perchlorate or $3,3^{\prime}$-dihexadecyloxacarbocyanine perchlorate according to the manufacturer's instructions (Molecular Probes, Eugene, OR, USA) and as previously described (32). Recipient mice were transfused via IV tail vein with $75 \mu \mathrm{L}$ of hGPA RBCs and a similar amount of wild-type RBCs. Survival of the transfused RBCs was determined by comparing the ratio of circulating hGPA RBCs to control RBCs in recipients longitudinally post-transfusion.

\section{Adoptive Transfer}

Single splenic cell suspensions from 8 to 10 weeks old female donor CD45.1 OT-II mice were prepared using gentle mechanical disruption, followed by RBC lysis with AcK buffer $(0.15 \mathrm{M}$ $\mathrm{NH} 4 \mathrm{Cl}, 10 \mathrm{mM} \mathrm{KHCO} 3$, and $0.1 \mathrm{mM}$ EDTA). CD4 ${ }^{+}$T-cells were isolated using a mouse $\mathrm{CD}^{+} \mathrm{T}$-cell-negative isolation selection kit (Stemcell Technologies, Vancouver, BC, Canada). Purified OTII CD4 ${ }^{+}$T-cells were retro-orbitally injected into recipient mice. Recipient C57BL/6 mice were transfused with HOD RBCs $24 \mathrm{~h}$ following adoptive transfer.

\section{Flow Cytometric Analysis RBC Flow Cytometric Crossmatch}

Levels of anti-hGPA/HOD alloantibodies in transfusion recipients were measured by a flow cytometric crossmatch assay as previously described (33) using IgM, total Igs, or IgG (BD Biosciences, San Jose, CA, USA). In brief, antigen-specific responses were determined by calculating an adjusted mean fluorescence intensity (MFI), which is the difference between the signal obtained with sera crossmatched with antigen-positive (hGPA/HOD) RBCs and that obtained with sera crossmatched with antigen-negative (FVB/NCr) RBCs. The adjusted MFI thus represents antibody (IgM, Igs, or IgG) that is specifically targeted against the non-self $\mathrm{RBC}$ antigen that the recipient was exposed to via transfusion. For the flow cytometric crossmatch assay, samples were analyzed on a four-color BD FACS Calibur or 8-color Miltenyi MACSQuant ${ }^{\circledR}$ Analyzer with analysis completed using Flo Jo software.

\section{Immune Cell Sub-Population Analysis}

To determine frequencies and numbers of different cell populations, flow cytometry was performed on single-cell suspensions from bone marrow (BM) and spleen tissues longitudinally, at specified time points. In brief, spleens were harvested and homogenized into a single-cell suspension in Hank's balanced salt solution (HBSS) using a 5-mL syringe plunger. Single cells from BM tissues were obtained by pipetting the tissue in and out several times in HBSS. For flow cytometric analysis of immune cells, RBCs were lysed using ammonium chloride and potassium bicarbonate salt solution. Cells were stained with different surface antibodies in buffer containing 0.1\% EDTA and $0.01 \%$ bovine serum albumin. Immune cell subsets in splenocytes and BM cells were analyzed via flow cytometry using fluorochrome-conjugated monoclonal antibodies to mouse surface markers CD19 (clone\#eBio1D3, eBiosciences), CD45R (B220, clone\#RA3-6B2, eBiosciences), CD5 (clone\#53-7.3, Biolegend), CD1d (clone\#1B1, Biolenged), GL7 (Clone\#GL7, Biolegend), CD95 (clone\#Jo2, BD pharmingen), CD138 (Clone\#281-2, Biolegend), TCR $\beta$ (Clone\#H57-597, Biolegend), CD4 (Clone\#GK1.5, Biolegend), CXCR5 (Clone\#2G8, BD pharmingen), PD1 (Clone\#29F.1A12, Biolegend), and BCL6 (Clone\#K112-91, BD pharmingen) (BD Biosciences, San Jose, CA, USA or eBiosciences San Diego, CA, USA or BioLegend San Diego, CA, USA). To detect regulatory T-cells (Tregs), intracellular Foxp3 staining with fix and permeabilization kit was performed (eBiosciences, San Diego, CA, USA) according to the manufacturer's instructions. Live cells were first gated using 
live/dead stain (BioLegend, San Diego, CA, USA). T-follicular helper $(\mathrm{TFH})$ cells were defined as $\mathrm{CD} 4^{+} \mathrm{CXCR} 5^{\text {hi }} \mathrm{PD}-1^{+}$or $\mathrm{CD} 4^{+} \mathrm{BCL}^{+}$. Samples were analyzed on a LSR II flow cytometer (BD Biosciences, San Jose, CA, USA) or on Miltenyi MACSQuant ${ }^{\circledR}$ Analyzer.

\section{Immunofluorescence}

For analysis of germinal centers (GCs), spleens were cryopreserved in optimal cutting temperature medium, sectioned on a cryostat, and stained. Frozen spleens were sectioned at $8-\mu \mathrm{m}$ thickness fixed in $4 \%$ paraformaldehyde (Electron Microscopy Sciences, Hatfield, PA, USA) washed in ice-cold $\mathrm{PBS}$, and stained at room temperature for $60 \mathrm{~min}$ with antiB220 (AF488), anti-CD4 (PE), and biotinylated PNA in 5\% fetal calf serum/PBS solution. After primary staining, the slides were washed in ice-cold PBS three times and stained with streptavidin AF647. In some circumstances, AF647-conjugated GL7 was used for GC staining instead of PNA and observed similar staining patterns. For consistency, near middle sections of the spleens were used for staining and were then analyzed using an automated wide-field microscope (Nikon Eclipse Ti) and CCD camera (Qimaging Retiga 2000R) with NIS elements software. Number of GC was counted in the entire field of view of the spleen sections.

\section{Statistics}

All statistical analyses were performed using Graph Pad Prism software (San Diego, CA, USA). The Mann-Whitney $U$ test was used to determine significant differences between two groups, and the Kruskal-Wallis with Dunn's post-test or a two-way ANOVA with Tukey's multiple comparisons test were used when comparing more than two groups as indicated. Error bars represent one $\mathrm{SD}$, and significance was determined by a $p$-value of $<0.05$.

\section{RESULTS}

\section{Anti-hGPA IgG Is Generated after RBC Transfusion in the Presence but Not the Absence of Poly IC}

Mice treated with poly IC approximately $4 \mathrm{~h}$ prior to hGPA RBC transfusion generated anti-hGPA IgG, whereas those transfused in the absence of poly IC did not form detectable anti-hGPA IgG at any evaluated time point (Figure 1A) (26).

We next sought to characterize GC formation and other immune cell responses in alloimmunized and non-alloimmunized animals. Splenic GC cells were not readily characterized in alloimmunized animals in this hGPA model by flow cytometry (Figure S1A in Supplementary Material). In parallel to the flow cytometric studies, we also imaged the spleen posttransfusion for well-defined GC areas. Consistent with the flow data, we found no statistically significant differences in the number of GCs between groups within the fields evaluated (Figure S1B in Supplementary Material). Additional studies were completed after repeat transfusion with no significant GC formation identified in alloimmunized animals (Figure S1C in Supplementary Material); antigen-specific B-cell evaluation completed using $\mathrm{RBC}$ membrane ghosts from hGPA donors also failed to show GC formation in this model (data not shown). Although it cannot be ruled out that antihGPA IgG is formed through a GC-independent pathway (34), our approach to detect GC B-cells (in the absence of being able to identify antigen-specific cells) in the hGPA system lacks sensitivity.

Along with GCs, other cells in the spleen and BM were characterized longitudinally after transfusion of hGPA RBCs in the presence or absence of poly IC treatment. The frequencies of total BM plasma cells (PCs) were not significantly different between mice transfused in the presence or absence of poly IC at any studied time point (Figure S2A in Supplementary Material). Foxp $3^{+}$Tregs as well as TFH cells (gated as CXCR5 ${ }^{\text {hi }} \mathrm{PD}^{+}$out of total TCR $\beta^{+} \mathrm{CD} 4^{+} \mathrm{T}$-cells) were evaluated at multiple time points (days 2-14) post-transfusion in the presence or absence of poly IC pretreatment; no differences in total cell numbers were observed between the groups at any studied time point (Figures S2B,C in Supplementary Material). Finally, CD5 ${ }^{+}$B-cells were evaluated, with no differences observed in gated cell numbers between any group studied (Figure S2D in Supplementary Material).

\section{Clearance of Transfused hGPA RBCs Occurs Only in Animals That Generate Anti-hGPA IgG}

Antibodies of the IgG class that are specific for glycophorin may be clinically significant in humans, and we hypothesized that the anti-hGPA IgG generated by animals treated with poly IC prior to $\mathrm{RBC}$ transfusion would also be capable of leading to premature $\mathrm{RBC}$ clearance in our murine model. To investigate RBC clearance patterns, we labeled hGPA RBCs with one lipophilic dye and labeled wild-type FVB RBCs with a different lipophilic dye; a mixture of RBCs was then transfused in alloimmunized or nonalloimmunized animals and the ratio of circulating labeled FVB to hGPA RBCs was evaluated longitudinally post-transfusion (Figure 1B). In alloimmunized mice, there was rapid preferential clearance of $70-90 \%$ of transfused hGPA RBCs within the first $24 \mathrm{~h}$, with almost all hGPA RBCs cleared by day 4 posttransfusion. In contrast, there was no preferential clearance of transfused hGPA RBCs in non-alloimmunized or naive mice by day 4 post-transfusion (Figure 1C) or beyond. These data highlight the clinical significance of the humoral immune response to hGPA RBCs in the presence of poly IC and also suggest the lack of "cellular immune"-mediated RBC clearance in animals that failed to generate an anti-hGPA IgG response after prior hGPA transfusion.

\section{Anti-hGPA IgG Formation Is Dependent on Recipient CD4 Cells}

To determine whether the formation of RBC alloantibodies in the hGPA system was dependent on CD4 cells, we treated mice with CD4-depleting monoclonal antibody GK1.5 or an isotypematched control antibody prior to and during primary transfusion with hGPA RBCs and poly IC (Figure S3 in Supplementary Material shows CD4 status at the time of transfusion). Anti-hGPA 
A

Anti-hGPA lgG

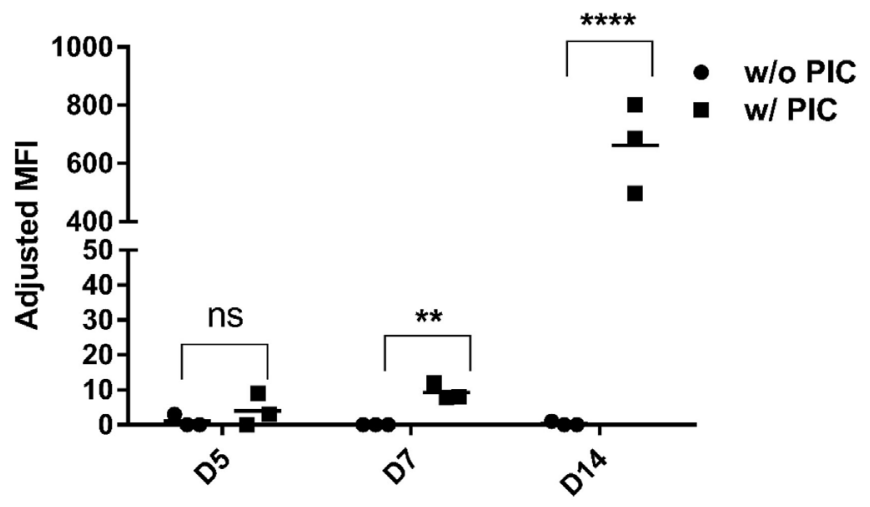

B
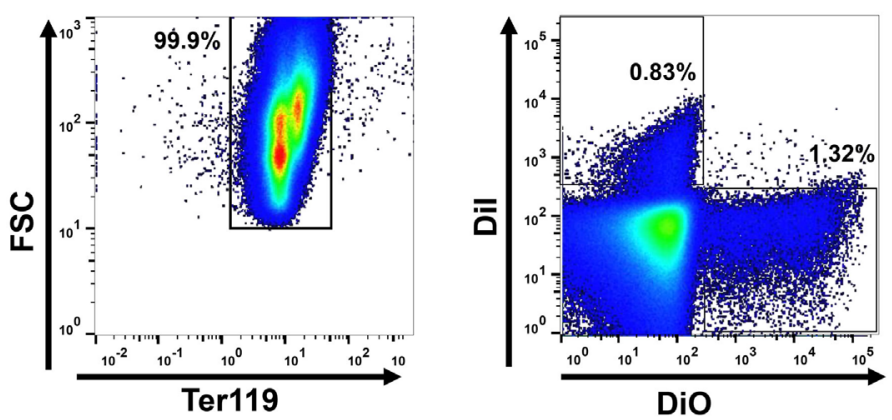

C

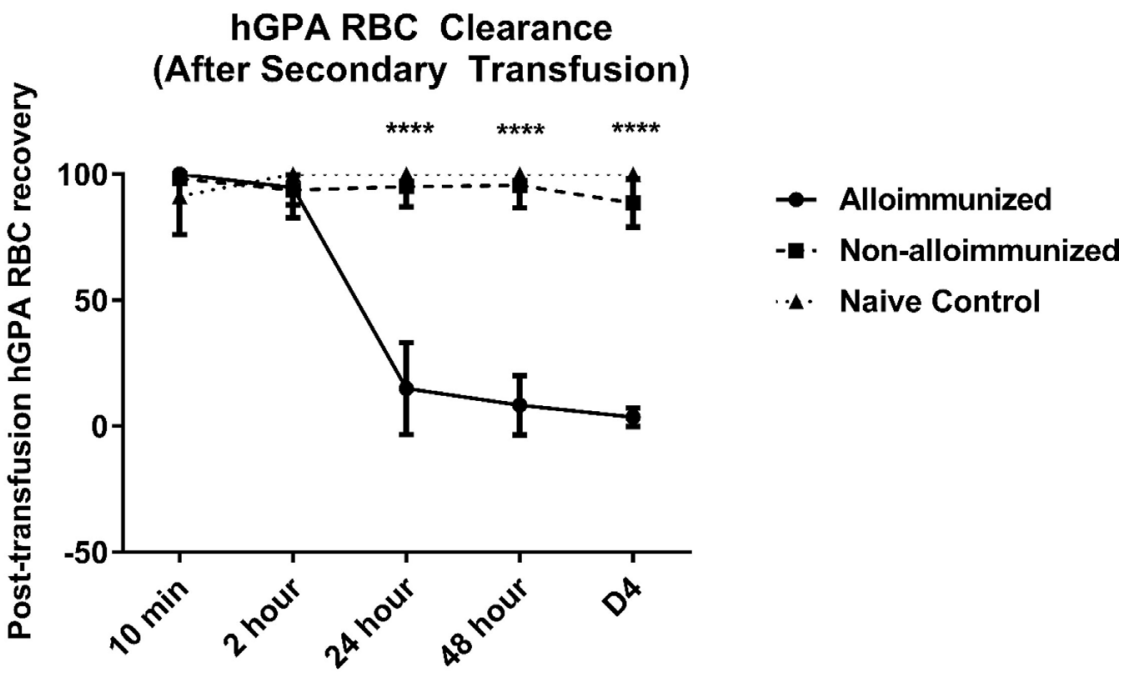

\section{Alloimmunized Non-alloimmunized Naive Control}

$\begin{array}{rlll}\text { Primary Transfusion } & \text { w/ PIC } & \text { w/o PIC } & \text { No transfusion } \\ \text { Secondary Transfusion } & \text { w/o PIC } & \text { w/o PIC } & \text { w/o PIC }\end{array}$

FIGURE 1 | Characterization of immune responses to transfused human glycophorin A (hGPA) red blood cells (RBCs), in the presence or absence of poly IC. (A) Serum anti-hGPA IgG at day 5 (D5), day 7 (D7), and day 14 (D14) post-transfusion represented as adjusted mean fluorescence intensity (MFI) in mice transfused with hPGA RBCs in the presence or absence of pretreatment with poly IC. (B) Alloimmunized animals (previously transfused with hGPA RBCs in the presence of poly IC) or non-alloimmunized (previously transfused without poly IC) were transfused for a second time with Dil-labeled syngeneic FVB RBCs and 3,3'-dihexadecyloxacarbocyanine perchlorate (DiO) labeled hGPA RBCs; representative plot showing the gating strategy for DiO and DiO-positive RBCs (pregated on Ter119+ cells). (C) Post-transfusion clearance curve in alloimmunized, non-alloimmunized, or naïve mice. Data are representative of at least two experiments ( $n=3-6$ mice per group per experiment). ${ }^{* *} p<0.01,{ }^{* \star \star *} p<0.0001$ determined by Mann-Whitney $U$ test in panel (A) and ANOVA in panel (C), between alloimmunized versus non-alloimmunized or naïve mice. There were no significant differences at any time point between naïve and non-alloimmunized mice. 
IgG was detectable only in isotype-matched control treated and not CD4-depleted animals on day 14 post-transfusion (Figure 2A).

Next, we sought to investigate the response to a secondary hGPA RBC challenge in mice previously transfused in a CD4depleted status. To do this, mice previously treated with GK1.5 or an isotype control antibody during the primary transfusion were rested for 3 weeks; the CD4 status at the time of primary and re-transfusion is shown in Figure S3 in Supplementary Material. The animals were then treated with poly IC and re-challenged with hGPA RBCs. Despite having CD4 T-cells present during this second hGPA RBC transfusion, mice that saw the hGPA antigen initially in a state of $\mathrm{CD} 4$ depletion remained non-responders to the secondary RBC exposure (Figure 2B). In contrast, the isotype control antibody-treated mice had a significant boostable antihGPA IgG response to the secondary transfusion.

Finally, we investigated the fate of transfused hGPA RBCs in these animals. There was essentially no preferential hGPA RBC clearance over co-transfused antigen-negative FVB RBCs in animals treated with GK1.5 prior to their initial but not secondary RBC transfusion. In contrast, approximately $90 \%$ of transfused hGPA RBCs cleared in the control group within the first $24 \mathrm{~h}$ post-transfusion, with almost all hGPA RBCs being cleared within 5 days post-transfusion (Figure 2C).

Taken together, these data suggest that poly IC-driven hGPA alloimmunization is CD4 dependent, that conditions around the initial hGPA RBC exposure dictate secondary responses (e.g., CD4 depletion at primary $\mathrm{RBC}$ exposure induces tolerance to secondary exposures), and that only animals with detectable anti-hGPA IgG have premature clearance of incompatible transfused RBCs.

\section{Blocking CD40L Signaling Abrogates the Formation of hGPA Alloantibodies}

Since CD40-CD40L signaling is critical in both antigen-presenting cell (APC):CD4 interactions (35) and T-cell:B-cell interactions $(36,37)$, we sought to determine if blocking this signaling could prevent hGPA RBC alloantibody formation. Mice were treated with the anti-CD40L monoclonal antibody, MR1, or an isotypematched control, prior to treatment with poly IC and transfusion with hGPA RBCs. MR1-treated mice failed to generate detectable anti-hGPA IgG (Figure 3A shows data from 14 days post-transfusion). As we observed with CD4-depleted animals, mice initially transfused in the presence of CD40L blockade failed to generate detectable anti-hGPA IgG when treated with poly IC and retransfused with hGPA 5 weeks later (Figure 3B). In contrast, the isotype control antibody-treated mice demonstrated a boostable anti-hGPA IgG response. These data suggest that the transfusion recipient's CD40/CD40L interaction at first hGPA RBC exposure dictates subsequent hGPA responses.

Next, splenic GC responses were evaluated in animals 8 days following their secondary RBC exposure. Animals transfused in the presence of MR1 had essentially no detectable GL7 ${ }^{+} \mathrm{CD} 95^{+}$ $\mathrm{B}$-cells (Figure 3C). In contrast, those transfused in the presence of an isotype type-matched controls had GCs (albeit no more than those found in naïve animals or in animals treated with poly IC alone).

\section{Non-Responsiveness to Subsequent hGPA RBC Exposure after CD40L Blockade Is Antigen Specific}

To investigate whether CD40L blockade during initial hGPA $\mathrm{RBC}$ exposure led to diffuse RBC non-responsiveness or to antigen-specific RBC non-responsiveness, we exposed alloimmunized and non-alloimmunized mice to a third-party antigen (KEL glycoprotein) on transfused RBCs. The immune response induced by the KEL-expressing RBCs used for these experiments (KEL ${ }^{\text {hi }} \mathrm{RBCs}$ ) is T-cell dependent (data not shown, manuscript in preparation). Serum was collected longitudinally posttransfusion, with flow cytometric crossmatch assays completed using transgenic KEL or wild-type RBCs as targets. MR1-treated mice, isotype control mice, and naïve WT mice had similar peak levels of anti-KEL antibodies (Figure 3D), suggesting antigenspecific non-responsiveness.

\section{CD40L Blockade Prevents Expansion of Antigen-Specific CD4 ${ }^{+}$T-Cells, Prevents GC Formation, and Blunts HOD RBC Alloantibody Responses}

To investigate the potential mechanisms of action of CD40L blockade using MR1 Ab in a transfusion setting, we turned to a transgenic murine model that enables the study of antigenspecific CD4 ${ }^{+}$T-cells. HOD transgenic mice have RBC-specific expression of the hen egg lysozyme, ovalbumin, and the human Duffy antigen, and OTII CD4 ${ }^{+}$T-cells have been shown to proliferate in recipients transfused with $\operatorname{HOD} \operatorname{RBCs}(12,38)$. For these experiments, naïve $\mathrm{C} 57 \mathrm{BL} / 6$ mice were adoptively transferred with 10,000 CD $45.1^{+}$OTII CD4 ${ }^{+}$T-cells and then transfused with HOD RBCs and poly IC with or without MR1 treatment. MR1treated mice demonstrated significant blunting of peak anti-HOD IgG responses compared to those transfused in the absence of MR1 treatment (Figure S4 in Supplementary Material).

To determine the effect of MR1 treatment on the expansion of antigen-specific T-cells, we evaluated the frequency of antigenspecific CD45.1+ OTII cells in transfusion recipients at multiple time points post-transfusion (Figure S4 in Supplementary Material and Figure 4A show representative gating). The frequency of OTII cells was significantly lower in the MR1-treated group compared to the isotype-matched control treated group on days 7 and 14 post-HOD transfusion (Figure 4A). Given prior studies of MR1 and Tregs in graft-versus-host disease (GVHD) models (39), we evaluated these cells in our transfused animals. We found no significant differences in the number of endogenous or antigenspecific (CD45.1 $1^{+}$OTII) Foxp $3^{+} \mathrm{CD}^{+}$Tregs between groups (Figure 4B), with there being extremely few antigen-specific OTII cells to characterize in animals treated with MR1. To further investigate whether Tregs may play a critical role in the mechanism of action of MR1 alloantibody mitigation, we depleted Tregs using PC61 antibody (BioXcell), treated animals with MR1, transfused with transgenic RBCs, and evaluated alloantibody response 2 weeks later. MR1 treatment prevented RBC alloimmunization in both Treg-depleted and Treg-replete mice in this pilot experiment (data not shown), suggesting that Treg expansion is not necessary 
A

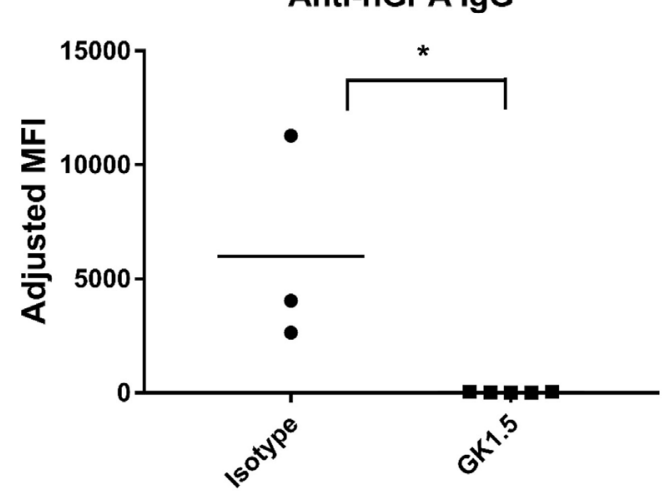

B

Anti-hGPA IgG after Primary or Secondary RBC Exposure

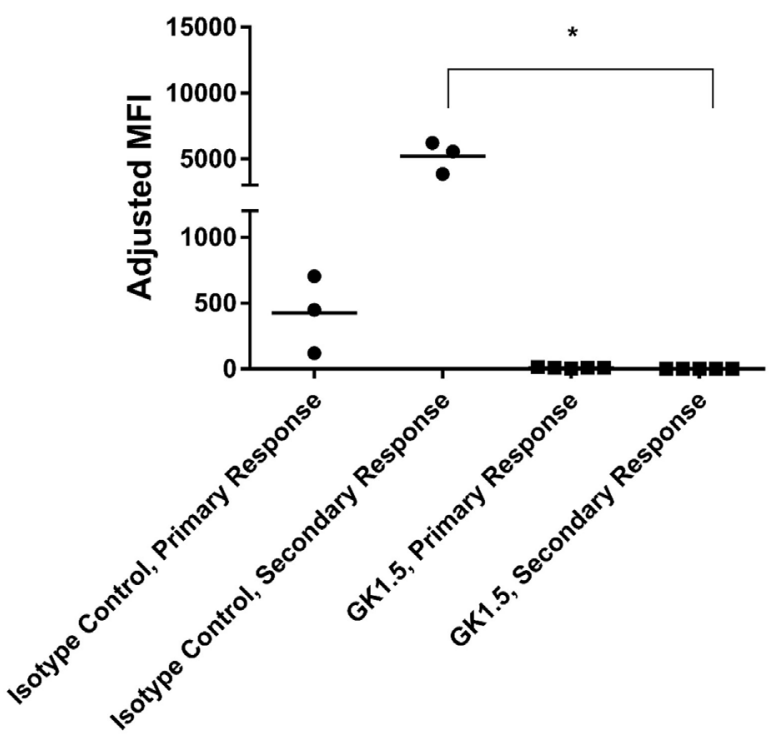

C

hGPA RBC Clearance (After Secondary Transfusion)

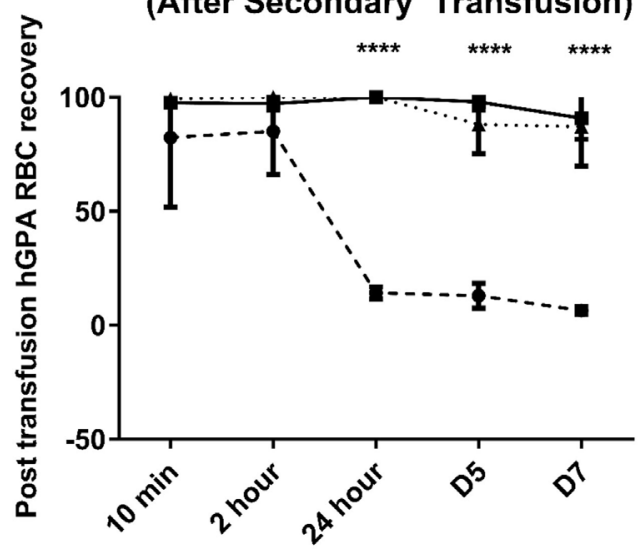

$\rightarrow$ GK1.5 treated

-๑. Isotype treated

A. Naive Control

FIGURE 2 | Alloantibody formation in the human glycophorin A (hGPA) RBC system is CD4 dependent. (A) Serum anti-hGPA IgG at day 14 represented as adjusted mean fluorescence intensity (MFI) in mice treated with GK1.5 or isotype-matched control antibody during primary transfusion. (B) Serum anti-hGPA IgG after primary or secondary hGPA RBC transfusion, with secondary transfusion given 5 weeks after primary transfusion and 3 weeks after last GK1.5 treatment. (C) Post-

transfusion RBC clearance curve in GK1.5-treated, isotype-matched control antibody-treated, or naïve mice after secondary transfusion. ${ }^{*} p<0.05$ determined by Mann-Whitney $U$ test. ${ }^{* \star \star *} p<0.0001$ determined by ANOVA between isotype control or naive and GK1.5-treated mice. There were no significant differences at any time point between naïve and isotype control mice. Data are representative of at least two experiments ( $n=3$ to 6 mice per group per experiment). 
A

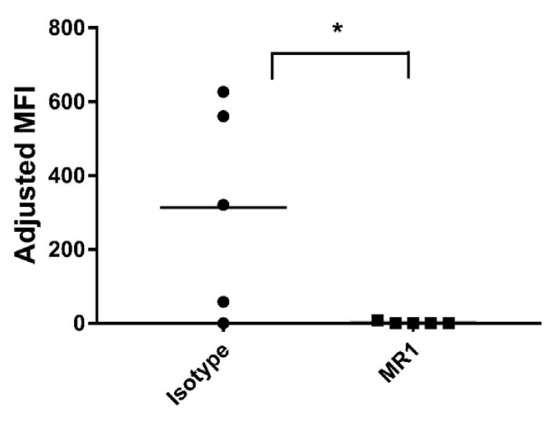

C
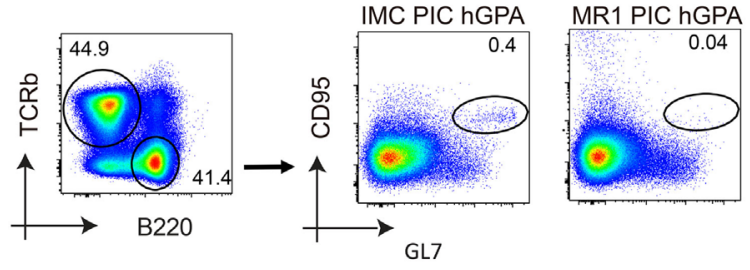

D

Response to 3rd Party Antigen (Anti-KEL IgG)
B
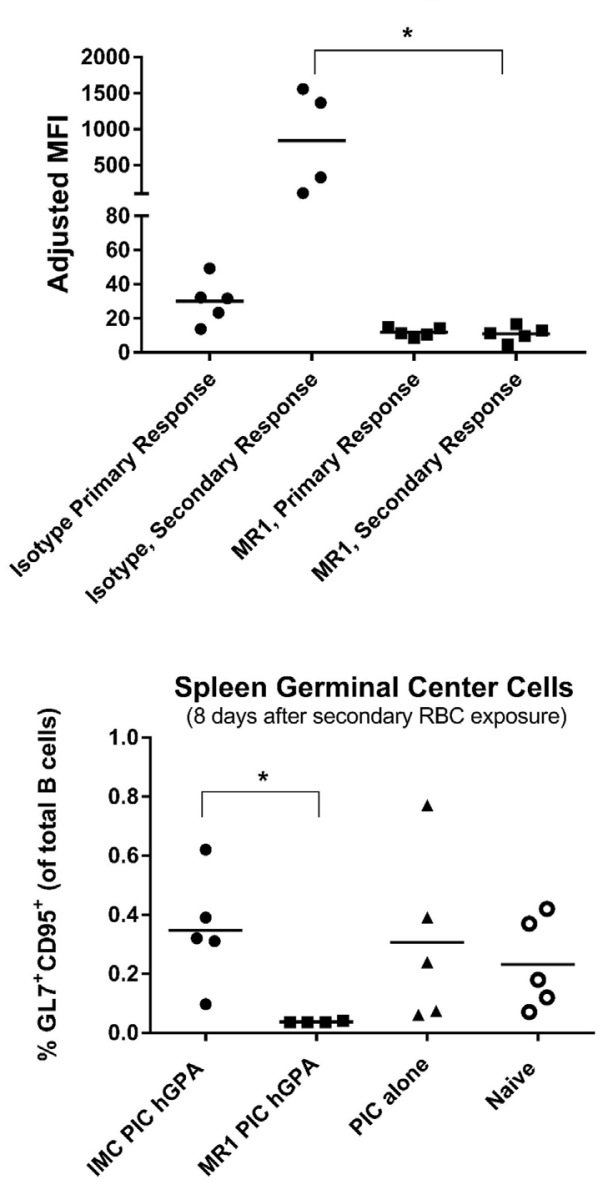

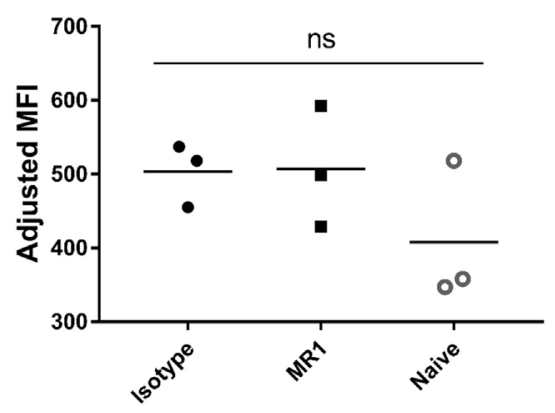

FIGURE 3 | Alloimmunization to human glycophorin A (hGPA) RBCs is abrogated upon blocking CD40L, a co-stimulatory molecule. (A) Serum anti-hGPA IgG at day 14 post-transfusion represented as adjusted mean fluorescence intensity (MFI) in mice treated with MR1 or isotype antibody during primary transfusion. (B) Serum anti-hGPA IgG after primary transfusion (7 days before secondary transfusion) and 14 days after secondary transfusion. (C) Splenic germinal centers (GL7+ CD95+ B-cells) 8 days after secondary RBC exposure. (D) Serum anti-KEL IgG after primary KEL transfusion in naiive animals or in those previously treated with MR1 or isotype-matched control antibody during prior hGPA transfusion. ${ }^{*} p<0.05$ determined by Mann-Whitney $U$ test or ANOVA. Data are representative of at least two experiments ( $n=3-5$ mice per group per experiment).

for the mechanism of action of MR1 in this system. We also evaluated endogenous and antigen-specific TFH cells in the transfused animals and found significantly decreased TFH cell numbers in animals treated with MR1 compared to those treated with the isotype-matched control (Figure 4C).
Finally, we evaluated splenic GC responses in these transfusion recipients. HOD RBCs were able to induce robust GC formation following a single transfusion. Treatment with MR1, however, fully prevented GC formation in transfused animals (Figures 5A,B). 


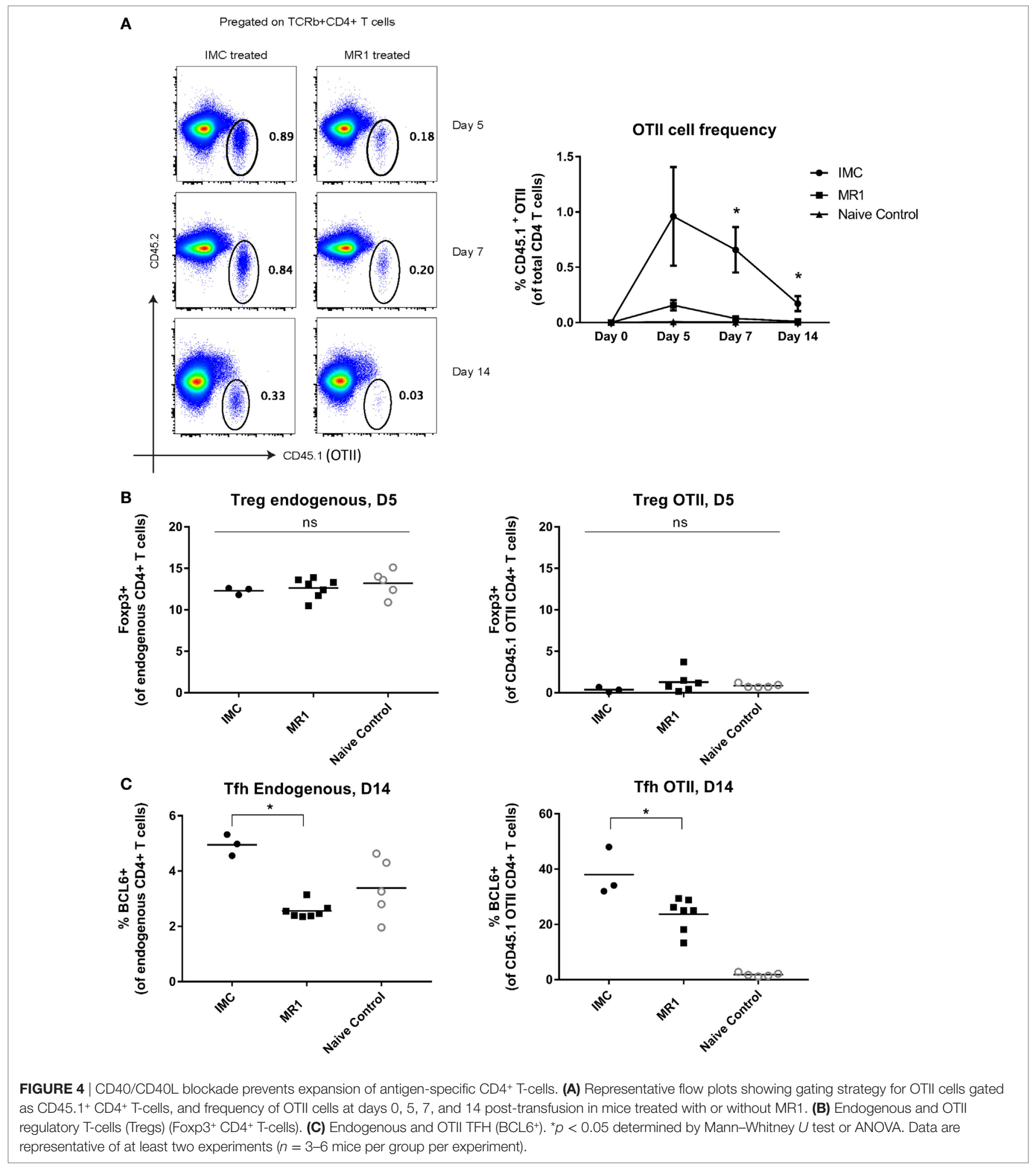

\section{DISCUSSION}

In this article, we have shown the importance of recipient $\mathrm{CD} 4^{+}$ cells and $\mathrm{CD} 40 \mathrm{~L}$ in the alloimmune response to transfused murine RBCs expressing the hGPA antigen. Poly IC or some adjuvant is required in this model to lead to an alloimmune response, which is consistent with data emerging in humans about a correlation between certain types of recipient inflammation and alloimmunization $(7,40)$. Notably, any initial hGPA RBC exposure that resulted in a non-detectable anti-hGPA humoral response 


\section{A Spleen Germinal Center Cells, D14}

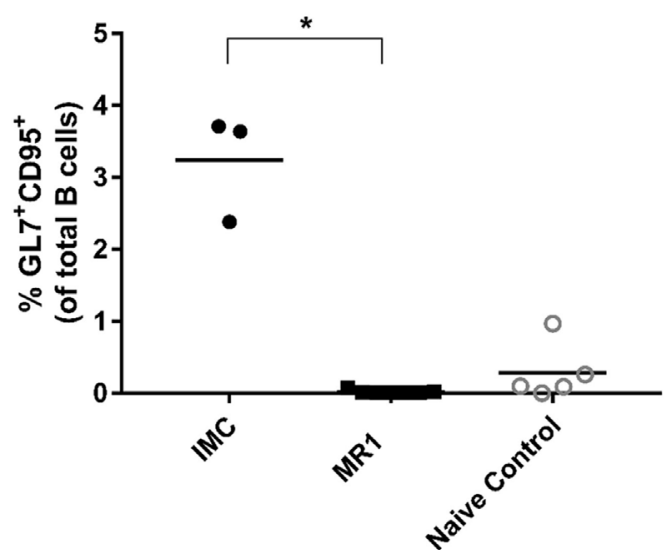

Spleen Germinal Center Cells, D14

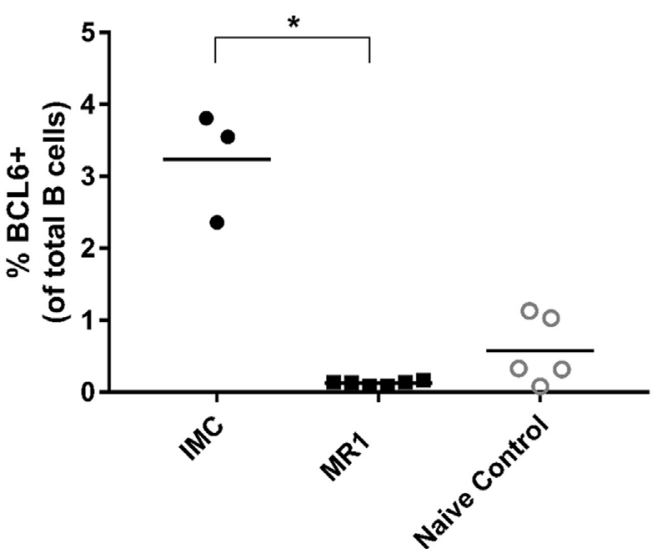

FIGURE 5 | CD40/CD40L blockade prevents germinal center formation after HOD (hen egg lysozyme, ovalbumin, and human duffy ${ }^{b}$ transfusion. (A) Splenic germinal centers (GL7+ CD95+ B-cells) or (B) BCL6+ B-cells cells, 14 days after primary HOD RBC exposure. ${ }^{*} p<0.05$ by ANOVA. Data are representative of two experiments ( $n=3-6$ mice per group per experiment).

appeared to tolerize the transfusion recipient in an antigenspecific manner, with future hGPA but not third-party antigen $\mathrm{RBC}$ exposures failing to induce detectable alloimmune responses.

The fact that an adjuvant is required in this model for a humoral immune response to occur, in combination with the fact that CD4 depletion or CD40L blockade can induce complete and long-lasting immune tolerance in an antigen-specific manner, suggest but do not conclusively confirm that immune responses to antigens on transfused RBCs are distinct from those to other antigens administered in a soluble form or through different routes. For example, although CD40L blockade has been shown to decrease skin and organ transplant rejection in mice and in non-human primates, suppression of the recipient $\mathrm{CD}^{+}$ T-cell component of transplant rejection may require additional co-stimulatory molecule blockade or immunosuppression $(39,41,42)$. Further, although CD40L blockade has been shown to prevent a primary immune response to factor VIII exposure in murine models of hemophilia (43), this non-responsiveness does not equate to long-lasting tolerance. By virtue of their circulation solely in the intravascular space and their sterile nature, RBCs may in fact be unique vehicles through which to induce tolerance (44). To date, there have been no studies in humans to determine whether non-responsiveness to RBC antigens (defined as the lack of a detectable anti-RBC alloantibody response after a transfusion) equates to tolerance or is simply due to ignorance. Data documenting low rates of RBC alloimmunization in chronically transfused patients with hemoglobinopathies initially exposed to RBCs at young ages (45-47) lend support to the idea of tolerance induction. Recent studies by Hubbell et al., comparing responses of soluble antigen to that of the same antigen associated with RBCs, demonstrate differences in the way the immune system views and responds to the same antigen presented in a different manner (48-50). These cited studies, undertaken with the hypothesis that RBC-surface bound antigen would be cleared tolerogenically along with eryptotic debris, also highlight the role of recipient T-cells in the tolerance induction process (49).
Our studies build upon the past work of responders/nonresponders to transfused $\mathrm{RBC}$ antigens published by others $(25,51,52)$. Given the lack of antigen-specific tools for studies in the hGPA system, we turned to the HOD model for mechanistic investigation. Our data using CD40L blockade in the HOD system demonstrated the prevention of antigen-specific $\mathrm{CD}^{+}$ T-cell expansion. Although an increased ratio of Tregs to Teff has been observed with CD40L blockade in murine GVHD models $(39,53)$, our data suggest that Tregs do not appear to be critically important to the prevention of HOD RBC alloimmunization by MR1 treatment.

In addition to impacting Treg/Teff ratios, studies in other models suggest that CD40L blockade also impacts APC cytokine production and/or downstream B-cell responses. This blockade has been shown to be important not only for cytokine production from APCs (31) but also for upregulation of ICAM-1, CD80, and CD86 on these cells (54). One cytokine of particular interest in $\mathrm{RBC}$ alloantibody responses is IL-6, with a recent manuscript describing the critical importance of IL- 6 and IL-6R $\alpha$ signaling on $\mathrm{CD}^{+} \mathrm{T}$-cells in the development of antibodies against HOD RBCs (38). Of further interest is the role that bridging channel dendritic cells play in immune responses to transfused RBCs, given the recently described role of $33 \mathrm{D} 1 \mathrm{CD} 4^{+}$conventional dendritic cells in mediating alloantibody responses to HOD RBCs (12). The CD40L signaling axis has been shown to be required for B-cell proliferation and survival and for sustaining TFH cells during GC responses (55). Consistent with this, we observed that MR1-treated animals had no GC formation in studies in the hGPA and HOD systems. Future studies investigating $\mathrm{CD}^{+}$ T-cell/B-cell interactions are warranted, with one unique aspect of antigens on RBCs being their ability to crosslink B-cell receptors. Studies completed in a different murine model have shown that the tolerance induced following transfusion of RBCs expressing the KEL glycoprotein at very low density (KEL ${ }^{\text {lo }} \mathrm{RBCs}$ ) can be adoptively transferred via B-cells from non-responder animals to naïve, irradiated, MuMT mice (56). These data highlight the 
potential importance of B-cells in RBC antigen-induced tolerance induction and maintenance.

Our studies focused on humoral alloimmunity, as alloantibodies are a measurable and clinically relevant immunologic outcome in transfusion medicine. However, it has recently been appreciated that recipient $\mathrm{CD}^{+}$T-cells may play a role in the antibodyindependent premature clearance of transfused platelets in a murine model (57). In our current study, we thus compared the clearance of transfused hGPA RBCs in non-alloimmunized and alloimmunized animals, as a first evaluation of whether cellular mediated immunity may have been induced in animals who lacked a detectable humoral immune response. No premature hGPA RBC clearance was observed after transfusion in any nonalloimmunized animal lacking detectable anti-hGPA alloantibodies. Despite this lack of an obvious "cellular immune response," it cannot be ruled out that transfusions even under "tolerogenic" conditions may have additional immunologic sequelae. For example, it is at least theoretically possible that transfusions may be able to induce cell-mediated immunity capable of priming a recipient for hematopoietic stem cell transplant rejection, with such rejection previously described in murine models involving platelet or RBC transfusions $(28,58)$.

We completed the described studies in the hGPA system, given the clinical relevance of this human blood group and the responder/non-responder status we observe in recipients transfused in the presence or absence of an adjuvant. However, the inability to evaluate hGPA antigen-specific T-cells or B-cells limits mechanistic study options in this system and also limits the value of descriptive characterizations of GCs, PCs, and the like. Another limitation includes the inability to study hGPA responses in animals genetically lacking cell subsets or pathways, as few such animals exist on the same genetic (FVB/H2-q) background. In this article, we describe non-responder mice as being tolerized, yet future studies in other model systems are needed to fully characterize which recipient cell subsets may be able to transfer tolerance to naïve recipients. We focused our co-stimulatory molecule studies on CD40L blockade, although this is but one of many co-stimulatory/co-inhibitory pathways to investigate. As limitations to the translation of any CD40L blockade therapy may involve the risk of thromboembolism due to CD154 expression on platelets (59), blocking therapies that do not directly impact CD40L are actively being investigated in other models (60).

\section{REFERENCES}

1. 2009 National Blood Collection and Utilization Survey Report. US Department of Health and Human Services (2011). Available from: http://www.hhs.gov/ ash/bloodsafety/nbcus/index.html

2. Hendrickson JE, Hillyer CD. Noninfectious serious hazards of transfusion. Anesth Analg (2009) 108:759-69. doi:10.1213/ane.0b013e3181930a6e

3. Savage WJ. Transfusion reactions. Hematol Oncol Clin North Am (2016) 30:619-34. doi:10.1016/j.hoc.2016.01.012

4. Reid M, Lomas-Francis C. The Blood Group Antigen Facts Book. Amsterdam: Elsevier Academic Press (2004).

5. Reid ME, Mohandas N. Red blood cell blood group antigens: structure and function. Semin Hematol (2004) 41:93-117. doi:10.1053/j.seminhematol. 2004.01.001

6. Hendrickson JE, Tormey CA. Red blood cell antibodies in hematology/ oncology patients: interpretation of immunohematologic tests and clinical
In conclusion, our data show the importance of recipient $\mathrm{CD}^{+}$cells and the CD40/CD40L interaction (potentially between APC:T-cells or T-cells:B-cells) in determining whether a recipient becomes alloimmunized or tolerized to antigens on transfused RBCs. Our studies lay the groundwork for future studies investigating responsiveness/non-responsiveness to transfused RBC antigens in animal models and in humans and for expanding studies evaluating RBCs as vehicles through which to induce tolerance.

\section{ETHICS STATEMENT}

All procedures and protocols were approved by Emory and Yale University's Institutional Animal Care and Use Committee.

\section{AUTHOR CONTRIBUTIONS}

PN, DL, SP, SS, and JH designed the experiments and performed the primary experiments; JL, MS, DG, and DM assisted with the experiments; PN, DL, and JH analyzed the results and made the figures; PN and JH wrote the manuscript; and all authors edited the manuscript.

\section{ACKNOWLEDGMENTS}

The authors would like to thank Dr. C. John Luckey and his laboratory members for helpful discussions.

\section{FUNDING}

This work was supported in part by a grant from the Emory Egleston Children's Center and a grant from the National Institutes of Health (R01 HL126076) to JH and a grant from the National Institutes of Health (T32 HL007974) to Brian Smith, Chair of the Department of Laboratory Medicine at Yale University School of Medicine.

\section{SUPPLEMENTARY MATERIAL}

The Supplementary Material for this article can be found online at http://journal.frontiersin.org/article/10.3389/fimmu.2017.00907/ full\#supplementary-material.

significance of detected antibodies. Hematol Oncol Clin North Am (2016) 30:635-51. doi:10.1016/j.hoc.2016.01.006

7. Fasano RM, Booth GS, Miles M, Du L, Koyama T, Meier ER, et al. Red blood cell alloimmunization is influenced by recipient inflammatory state at time of transfusion in patients with sickle cell disease. Br J Haematol (2015) 168:291-300. doi:10.1111/bjh.13123

8. Ryder AB, Hendrickson JE, Tormey CA. Chronic inflammatory autoimmune disorders are a risk factor for red blood cell alloimmunization. Br J Haematol (2016) 174:483-5. doi:10.1111/bjh.13781

9. Fatalities Reported to the FDA Following Blood Collection. US Department of Health and Human Services (FDA) (2014). Available from: http://www.fda. gov/BiologicsBloodVaccines/SafetyAvailability

10. Nickel RS, Hendrickson JE, Fasano RM, Meyer EK, Winkler AM, Yee MM, et al. Impact of red blood cell alloimmunization on sickle cell disease mortality: a case series. Transfusion (2016) 56:107-14. doi:10.1111/ trf.13379 
11. Gardner K, Hoppe C, Mijovic A, Thein SL. How we treat delayed haemolytic transfusion reactions in patients with sickle cell disease. Br J Haematol (2015) 170:745-56. doi:10.1111/bjh.13494

12. Calabro S, Gallman A, Gowthaman U, Liu D, Chen P, Liu J, et al. Bridging channel dendritic cells induce immunity to transfused red blood cells. J Exp Med (2016) 213:887-96. doi:10.1084/jem.20151720

13. Hendrickson JE, Delaney M. Hemolytic disease of the fetus and newborn: modern practice and future investigations. Transfus Med Rev (2016) 30:159-64. doi:10.1016/j.tmrv.2016.05.008

14. Webb J, Abraham A. Complex transfusion issues in pediatric hematopoietic stem cell transplantation. Transfus Med Rev (2016) 30:202-8. doi:10.1016/j. tmrv.2016.06.002

15. Lerut E, Van Damme B, Noizat-Pirenne F, Emonds MP, Rouger P, Vanrenterghem Y, et al. Duffy and Kidd blood group antigens: minor histocompatibility antigens involved in renal allograft rejection? Transfusion (2007) 47:28-40. doi:10.1111/j.1537-2995.2007.01060.x

16. Rourk A, Squires JE. Implications of the Kidd blood group system in renal transplantation. Immunohematology (2012) 28:90-4.

17. Chou ST, Fasano RM. Management of patients with sickle cell disease using transfusion therapy: guidelines and complications. Hematol Oncol Clin North Am (2016) 30:591-608. doi:10.1016/j.hoc.2016.01.011

18. Chou ST, Jackson T, Vege S, Smith-Whitley K, Friedman DF, Westhoff CM. High prevalence of red blood cell alloimmunization in sickle cell disease despite transfusion from Rh-matched minority donors. Blood (2013) 122:1062-71. doi:10.1182/blood-2013-03-490623

19. Fasano RM, Chou ST. Red blood cell antigen genotyping for sickle cell disease, thalassemia, and other transfusion complications. Transfus Med Rev (2016) 30:197-201. doi:10.1016/j.tmrv.2016.05.011

20. Osby M, Shulman IA. Phenotype matching of donor red blood cell units for nonalloimmunized sickle cell disease patients: a survey of 1182 North American laboratories. Arch Pathol Lab Med (2005) 129:190-3.

21. Kacker S, Ness PM, Savage WJ, Frick KD, Shirey RS, King KE, et al. Economic evaluation of a hypothetical screening assay for alloimmunization risk among transfused patients with sickle cell disease. Transfusion (2014) 54:2034-44. doi: $10.1111 /$ trf. 12585

22. Ryder AB, Zimring JC, Hendrickson JE. Factors influencing RBC alloimmunization: lessons learned from murine models. Transfus Med Hemother (2014) 41:406-19. doi:10.1159/000368995

23. Noizat-Pirenne F, Tournamille C, Bierling P, Roudot-Thoraval F, Le Pennec PY, Rouger P, et al. Relative immunogenicity of Fya and $\mathrm{K}$ antigens in a Caucasian population, based on HLA class II restriction analysis. Transfusion (2006) 46:1328-33. doi:10.1111/j.1537-2995.2006.00900.x

24. Auffray I, Marfatia S, De Jong K, Lee G, Huang CH, Paszty C, et al. Glycophorin A dimerization and band 3 interaction during erythroid membrane biogenesis: in vivo studies in human glycophorin A transgenic mice. Blood (2001) 97:2872-8. doi:10.1182/blood.V97.9.2872

25. Yu J, Heck S, Yazdanbakhsh K. Prevention of red cell alloimmunization by CD25 regulatory T cells in mouse models. Am J Hematol (2007) 82:691-6. doi:10.1002/ajh.20959

26. Smith NH, Hod EA, Spitalnik SL, Zimring JC, Hendrickson JE. Transfusion in the absence of inflammation induces antigen-specific tolerance to murine RBCs. Blood (2012) 119:1566-9. doi:10.1182/blood-2011-09-382655

27. Gibb DR, Liu J, Natarajan P, Santhanakrishnan M, Madrid DJ, Eisenbarth SC, et al. Type I IFN is necessary and sufficient for inflammationinduced red blood cell alloimmunization in mice. J Immunol (2017). doi:10.4049/jimmunol.1700401

28. Desmarets M, Cadwell CM, Peterson KR, Neades R, Zimring JC. Minor histocompatibility antigens on transfused leukoreduced units of red blood cells induce bone marrow transplant rejection in a mouse model. Blood (2009) 114:2315-22. doi:10.1182/blood-2009-04-214387

29. Smith NH, Henry KL, Cadwell CM, Bennett A, Hendrickson JE, Frame T, et al. Generation of transgenic mice with antithetical KEL1 and KEL2 human blood group antigens on red blood cells. Transfusion (2012) 52:2620-30. doi:10.1111/j.1537-2995.2012.03641.x

30. Gilson CR, Zimring JC. Alloimmunization to transfused platelets requires priming of CD4+ T cells in the splenic microenvironment in a murine model. Transfusion (2012) 52:849-59. doi:10.1111/j.1537-2995.2011.03346.x

31. Ferrer IR, Liu D, Pinelli DF, Koehn BH, Stempora LL, Ford ML. CD40/CD154 blockade inhibits dendritic cell expression of inflammatory cytokines but not costimulatory molecules. J Immunol (2012) 189:4387-95. doi:10.4049/ jimmunol.1201757

32. Liepkalns JS, Hod EA, Stowell SR, Cadwell CM, Spitalnik SL, Zimring JC. Biphasic clearance of incompatible red blood cells through a novel mechanism requiring neither complement nor Fcgamma receptors in a murine model. Transfusion (2012) 52:2631-45. doi:10.1111/j.1537-2995.2012.03647.x

33. Hendrickson JE, Desmarets M, Deshpande SS, Chadwick TE, Hillyer CD, Roback JD, et al. Recipient inflammation affects the frequency and magnitude of immunization to transfused red blood cells. Transfusion (2006) 46:1526-36. doi:10.1111/j.1537-2995.2006.00946.x

34. Takemori T, Kaji T, Takahashi Y, Shimoda M, Rajewsky K. Generation of memory B cells inside and outside germinal centers. Eur J Immunol (2014) 44:1258-64. doi:10.1002/eji.201343716

35. Cella M, Scheidegger D, Palmer-Lehmann K, Lane P, Lanzavecchia A, Alber G. Ligation of CD40 on dendritic cells triggers production of high levels of interleukin-12 and enhances T cell stimulatory capacity: T-T help via APC activation. J Exp Med (1996) 184:747-52. doi:10.1084/jem.184.2.747

36. Klaus SJ, Pinchuk LM, Ochs HD, Law CL, Fanslow WC, Armitage RJ, et al. Costimulation through CD28 enhances T cell-dependent B cell activation via CD40-CD40L interaction. J Immunol (1994) 152:5643-52.

37. Elgueta R, Benson MJ, De Vries VC, Wasiuk A, Guo Y, Noelle RJ. Molecular mechanism and function of CD40/CD40L engagement in the immune system. Immunol Rev (2009) 229:152-72. doi:10.1111/j.1600-065X.2009.00782.x

38. Arneja A, Salazar JE, Jiang W, Hendrickson JE, Zimring JC, Luckey CJ. Interleukin-6 receptor-alpha signaling drives anti-RBC alloantibody production and T-follicular helper cell differentiation in a murine model of red blood cell alloimmunization. Haematologica (2016) 101:e440-4. doi:10.3324/haematol.2016.149278

39. Vogel I, Verbinnen B, Van Gool S, Ceuppens JL. Regulatory T cell-dependent and -independent mechanisms of immune suppression by CD28/B7 and CD40/CD40L costimulation blockade. J Immunol (2016) 197:533-40. doi:10.4049/jimmunol.1502039

40. Evers D, Van Der Bom JG, Tijmensen J, Middelburg RA, De Haas M, Zalpuri S, et al. Red cell alloimmunisation in patients with different types of infections. Br J Haematol (2016) 175:956-66. doi:10.1111/bjh.14307

41. Larsen CP, Alexander DZ, Hollenbaugh D, Elwood ET, Ritchie SC, Aruffo A, et al. CD40-gp39 interactions play a critical role during allograft rejection. Suppression of allograft rejection by blockade of the CD40-gp39 pathway. Transplantation (1996) 61:4-9. doi:10.1097/00007890-19960115000002

42. Kirk AD, Burkly LC, Batty DS, Baumgartner RE, Berning JD, Buchanan K, et al. Treatment with humanized monoclonal antibody against CD154 prevents acute renal allograft rejection in nonhuman primates. Nat Med (1999) 5:686-93. doi:10.1038/9536

43. Reipert BM, Sasgary M, Ahmad RU, Auer W, Turecek PL, Schwarz HP. Blockade of CD40/CD40 ligand interactions prevents induction of factor VIII inhibitors in hemophilic mice but does not induce lasting immune tolerance. Thromb Haemost (2001) 86:1345-52.

44. Cremel M, Guerin N, Horand F, Banz A, Godfrin Y. Red blood cells as innovative antigen carrier to induce specific immune tolerance. Int J Pharm (2013) 443:39-49. doi:10.1016/j.ijpharm.2012.12.044

45. Spanos T, Karageorga M, Ladis V, Peristeri J, Hatziliami A, Kattamis C. Red cell alloantibodies in patients with thalassemia. Vox Sang (1990) 58:50-5. doi:10.1111/j.1423-0410.1990.tb02055.x

46. Tatari-Calderone Z, Minniti CP, Kratovil T, Stojakovic M, Vollmer A, Barjaktarevic I, et al. rs660 polymorphism in Ro52 (SSA1; TRIM21) is a marker for age-dependent tolerance induction and efficiency of alloimmunization in sickle cell disease. Mol Immunol (2009) 47:64-70. doi:10.1016/j. molimm.2008.12.027

47. Vichinsky E, Neumayr L, Trimble S, Giardina PJ, Cohen AR, Coates T, et al. Transfusion complications in thalassemia patients: a report from the Centers for Disease Control and Prevention (CME). Transfusion (2014) 54:972-81; quiz 971. doi:10.1111/trf.12348

48. Kontos S, Kourtis IC, Dane KY, Hubbell JA. Engineering antigens for in situ erythrocyte binding induces T-cell deletion. Proc Natl Acad Sci U S A (2013) 110:E60-8. doi:10.1073/pnas.1216353110

49. Grimm AJ, Kontos S, Diaceri G, Quaglia-Thermes X, Hubbell JA. Memory of tolerance and induction of regulatory $\mathrm{T}$ cells by erythrocyte-targeted antigens. Sci Rep (2015) 5:15907. doi:10.1038/srep15907 
50. Lorentz KM, Kontos S, Diaceri G, Henry H, Hubbell JA. Engineered binding to erythrocytes induces immunological tolerance to E. coli asparaginase. Sci Adv (2015) 1:e1500112. doi:10.1126/sciadv.1500112

51. Bao W, Yu J, Heck S, Yazdanbakhsh K. Regulatory T-cell status in red cell alloimmunized responder and nonresponder mice. Blood (2009) 113:5624-7. doi:10.1182/blood-2008-12-193748

52. Bao W, Zhong H, Li X, Lee MT, Schwartz J, Sheth S, et al. Immune regulation in chronically transfused allo-antibody responder and nonresponder patients with sickle cell disease and beta-thalassemia major. Am J Hematol (2011) 86:1001-6. doi:10.1002/ajh.22167

53. Verbinnen B, Billiau AD, Vermeiren J, Galicia G, Bullens DM, Boon L, et al. Contribution of regulatory $\mathrm{T}$ cells and effector $\mathrm{T}$ cell deletion in tolerance induction by costimulation blockade. J Immunol (2008) 181:1034-42. doi:10.4049/jimmunol.181.2.1034

54. Marigo I, Zilio S, Desantis G, Mlecnik B, Agnellini AH, Ugel S, et al. T cell cancer therapy requires CD40-CD40L activation of tumor necrosis factor and inducible nitric-oxide-synthase-producing dendritic cells. Cancer Cell (2016) 30:377-90. doi:10.1016/j.ccell.2016.08.004

55. Baumjohann D, Preite S, Reboldi A, Ronchi F, Ansel KM, Lanzavecchia A, et al. Persistent antigen and germinal center B cells sustain $\mathrm{T}$ follicular helper cell responses and phenotype. Immunity (2013) 38:596-605. doi:10.1016/j. immuni.2012.11.020

56. Arthur CM, Patel SR, Smith NH, Bennett A, Kamili NA, Mener A, et al. Antigen density dictates immune responsiveness following red blood cell transfusion. J Immunol (2017) 198:2671-80.
57. Arthur CM, Patel SR, Sullivan HC, Winkler AM, Tormey CA, Hendrickson JE, et al. CD8+ T cells mediate antibody-independent platelet clearance in mice. Blood (2016) 127:1823-7. doi:10.1182/blood-2015-10-673426

58. Patel CC Sr, Medford A, Zimring JC. Transfusion of minor histocompatibility antigen-mismatched platelets induces rejection of bone marrow transplants in mice. JClin Invest (2009) 119:2787-94. doi:10.1172/ JCI39590

59. Kawai T, Andrews D, Colvin RB, Sachs DH, Cosimi AB. Thromboembolic complications after treatment with monoclonal antibody against CD40 ligand. Nat Med (2000) 6:114. doi:10.1038/72162

60. Pinelli DF, Ford ML. Novel insights into anti-CD40/CD154 immunotherapy in transplant tolerance. Immunotherapy (2015) 7:399-410. doi:10.2217/ imt. 15.1

Conflict of Interest Statement: The authors declare that the research was conducted in the absence of any commercial or financial relationships that could be construed as a potential conflict of interest.

Copyright (C) 2017 Natarajan, Liu, Patel, Santhanakrishnan, Beitler, Liu, Gibb, Liepkalns, Madrid, Eisenbarth, Stowell and Hendrickson. This is an open-access article distributed under the terms of the Creative Commons Attribution License (CC $B Y)$. The use, distribution or reproduction in other forums is permitted, provided the original author(s) or licensor are credited and that the original publication in this journal is cited, in accordance with accepted academic practice. No use, distribution or reproduction is permitted which does not comply with these terms. 